\title{
A multi-layered risk exposure assessment approach for the shipping industry
}

\author{
Stephen Vander Hoorn ${ }^{1}$, Sabine Knapp ${ }^{2}$ \\ Econometric Institute, Erasmus University Rotterdam
}

Econometric Institute Report 2014-14

\begin{abstract}
Shipping activity has increased worldwide and maritime administrations are trying to enhance risk mitigation strategies by using proactive approaches. We present and discuss a conceptual framework to minimize potential harm based on a multi-layered approach which can be implemented in either real time for operational purposes or in prediction mode for medium or longer term strategic planning purposes. We introduce the concept of total risk exposure which integrates risk at the individual ship level with vessel traffic densities and location specific parameters such as weather and oceanographic conditions, geographical features or environmental sensitivities. A comprehensive and robust method to estimate and predict risk exposure can be beneficial to maritime administrations to enhance mitigation strategies and understand uncertainties. We further provide a proof of concept based on 53 million observations of vessel positions and individual risk profiles of 8,900 individual ships. We present examples on how endpoints can be visualized for two integrated risk layers - ship specific risk and vessel traffic densities. We further identify and discuss uncertainties and present our ideas on how other risk layers could be integrated in the future.
\end{abstract}

Keywords: Total risk exposure, binary logistic regression, spatial statistics, incident models, uncertainties, monetary value at risk

\footnotetext{
${ }^{1}$ Corresponding author address: Department of Mathematics and Statistics, University of Melbourne; vander@unimelb.edu.au

${ }^{2}$ Econometric Institute, Erasmus School of Economics, Erasmus University Rotterdam, P.O. Box 1738, 3000 DR Rotterdam, Netherlands; knapp@ese.eur.nl, Disclaimer: The views expressed in this article represent those of the author and do not necessarily represent those of the Australian Maritime Safety Authority (AMSA).
} 


\section{Introduction}

Most global trade is carried by sea, and shipping activity has increased by more than $300 \%$ since 1970 (UNCTAD, 2011). Increased shipping activity can pose potential harm to property, life and the marine environment. The goal of any regulatory authority at the port state or coastal level facing increased traffic is to mitigate potential harm and to gain a better understanding of or quantify uncertainties. Current approaches found in shipping show some limitations. Individual safety qualities of vessels are not considered and all vessels are treated equally which is unrealistic given that safety quality at the ship level can vary considerably (Heij and Knapp 2012, Heij et al. 2011, Knapp 2006). Underlying location specific environmental criteria such as the effect of wind, wave and currents are omitted due to the complexities involved in modelling their effect. Alternative approaches do not allow a dynamic or semi-dynamic implementation for a specific area with flexible spatial resolution. Furthermore, uncertainties in the estimates are not identified nor quantified (Merrick and van Dorp, 2006).

We propose an alternative conceptual framework to facilitate maritime risk assessment which may also assist to mitigate potential harm. The basic idea employed here is that total risk exposure (potential harm) can be best described as the combination of risk layers as follows: 1) ship specific risk as proxy to safety quality, 2) vessel traffic densities, 3) location specific physical environmental parameters such as wind, wave, currents and bathymetry, 4) other environmental factors such as sensitivities to pollution; and 5) intervention effects of risk control options (RCO) which can be deployed to mitigate risk (Figure 1). We define total risk exposure as the integration of the above described risk layers into a set of metrics given a spatial area. Each maritime administration can choose from a set of RCO to mitigate total risk exposure to an acceptable level of residual risk. In this context, we do not consider the effect of wind, waves and currents at the individual ship level (Heij and Knapp, 2014) due to the complexities involved with integrating these components into safety quality aspects of a particular vessel but we endeavor to account for the combined effect of the physical environmental layer on specific incidents such as drift groundings.

There are many types of risk exposure endpoints one could consider such as expected numbers of incidents, oil on water or on the coast or damages quantified in monetary terms. Due to the complexities involved in modelling physical environmental criteria (currents, waves and wind) and their combined effect towards drifting of large vessels or oil trajectories, we excluded this layer for now but its importance is recognized in the general framework presented here and is subject to additional research currently being carried out. We also briefly refer to use of expert knowledge for 
layers related to quantifying the effect of environmental sensitivities or the effect of risk control options by means of expert knowledge which are part of the conceptual framework. We feel that implementation aspects are important if used in practice by a regulatory authority. As such, the proposed method puts an emphasis on application aspects in real time (for operational vessel traffic management) or in prediction mode (for long term strategic planning) in the future.

Figure 1: Conceptual framework of the multi-layered risk approach

\section{RISK LAYERS (FACTORS):}

Layer 1: Ship specific risk (as proxy to safety quality)

Layer 2: Vessel traffic densities (e.g. nautical miles travelled, days at sea, collision candidates)

Layer 3: Physical environmental layer (currents, wind, waves, bathymetry, other geographical conditions such as difficult areas to navigate)

Layer 4: Environmental sensitivities (social, cultural, economics, ecological)

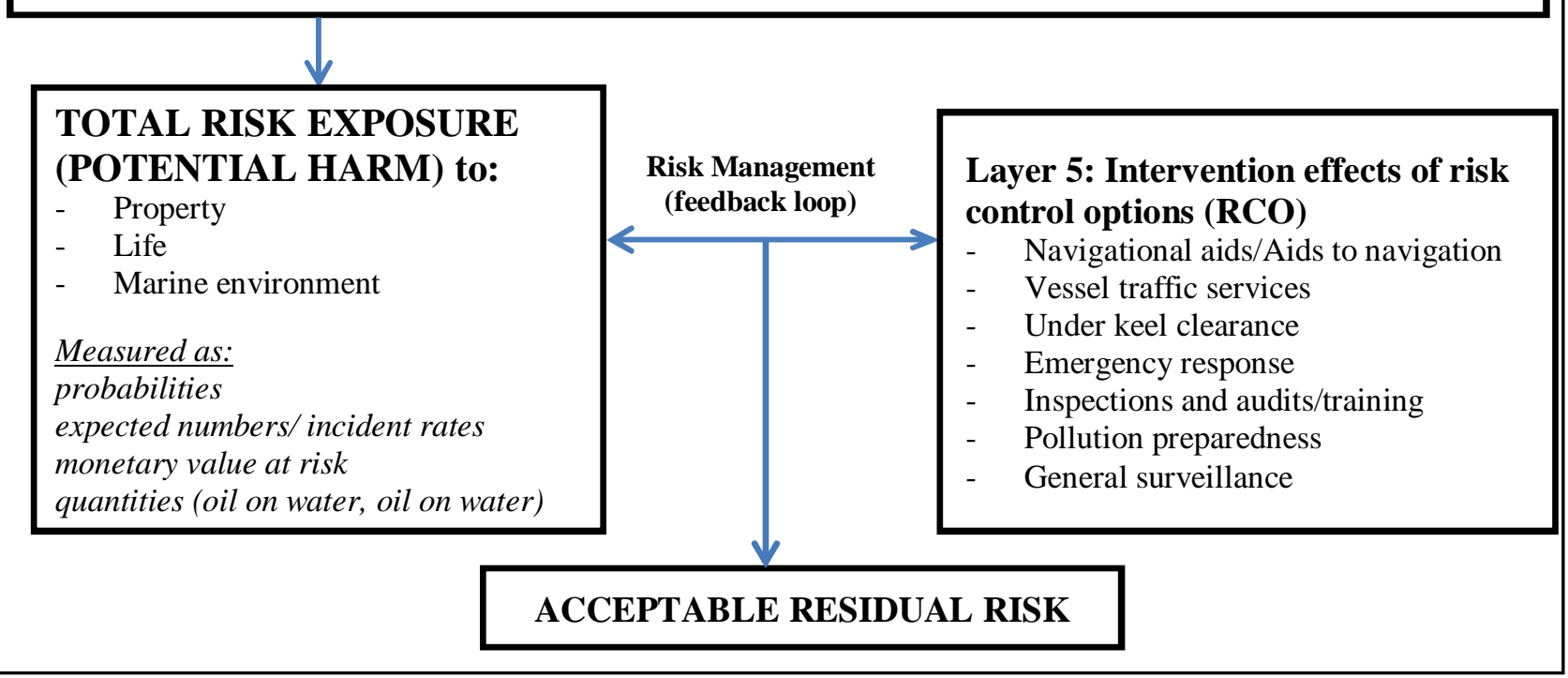

One major difference to other approaches is that in combining the first two layers, we capture the individual safety quality of a vessel in addition to vessel traffic densities rather than estimating risk based on the geometry of vessel traffic. It is believed that $80 \%$ of all navigational incidents are somehow related to human error (Hansen, 2007), hence the safety quality of a particular vessel is assumed to be more important in most incident cases. The proposed approach can be extended to other types of incidents beyond to what is presented here.

Besides discussing the conceptual framework, we also provide a proof of concept for the integration of the two first risk layers - that is ship specific risk with traffic densities based on a pilot project conducted in collaboration with the Australian Maritime Safety Authority (AMSA). In the pilot, we also included one of the risk control options in place (port state control inspections which can be quantified using quantitative methods) based on (Knapp et al. 2011, Bijwaard and Knapp 2009) and demonstrate the intervention effect on total risk exposure. In addition, we consider two endpoints - 
that is expected number of incidents and the quantification of damages in monetary terms based on (Heij and Knapp 2012). We denote the latter as monetary value at risk (MVR) which is estimated at the ship level and in aggregated form can be interpreted as risk exposure (proxy to potential harm) to be mitigated by a maritime administration excluding damages to the marine ecology or damages to cultural heritage which cannot be quantified in monetary terms.

We first discuss the theoretical framework and provide a methodology to estimate total risk exposure. We then present a simple proof of concept to demonstrate application aspects. Our conclusions will provide recommendations for future research to extend the approach further and add additional risk layers.

\section{Theoretical framework to estimate total risk exposure}

\subsection{Basic concepts}

Spatial analysis methods are utilised in many applications (de Smith MJ et al., 2013) but are not routinely used in the context of maritime risk assessment. The availability and quality of data dictates the degree to which reliable spatial analyses can be carried out. However, these techniques are specifically designed to evaluate where events occur and may be very useful in the analysis of incidence probabilities as they relate to traffic densities.

Aggregated statistical summaries of incident data provide a starting point to introduce the general concept and also to provide a set of definitions. Let's say historical data reveal that we have observed 10 serious collisions within a study region of interest over a 5 year period. If our study area is (say) 3 million square nautical miles, a very basic estimator of the intensity rate would be 2 per 3,000,000 $\mathrm{nm}^{2}$ per year. Of course, we would naturally want to know much more than this including: how precise is this estimate; how can this estimate be used to predict what will happen next year; does this rate differ by sub-region; what are the underlying factors causing (or associated with) the intensity rate, etc. The type of data behind this simple example above is referred to as spatial point pattern data and the corresponding statistical measure is referred to as the intensity of a point process. Intensity is the average density of points or expected number of points per unit area which may be either constant (homogenous) or may vary from location to location (inhomogenous). Investigation of the intensity is one of the first steps in analysing point pattern data. Formal definitions and techniques for investigating the intensity, have been well established (Badderly, 2010) and are included here in order to set the scene. 
More generally, the study area is a compact region $\boldsymbol{W} \subset \mathbb{R}^{2}$ in which we observe a realisation of a spatial point process $\boldsymbol{X}$. Our main interest is to determine the expected number of points from $X$ that would be expected to fall into $W$ or more generally, any sub-region $\boldsymbol{B}$ of $W, E[N(X \cap B)]$. For a homogenous point process $X$, the expected number of points in $B$ is proportional to the area of $B$ :

$$
E[N(X \cap B)]=\lambda \operatorname{area}(B)
$$

where the constant of proportionality $\lambda$ is the intensity. Intensity units are numbers per unit area $\left(\right.$ length ${ }^{-2}$ ). In the simplified case of a homogeneous point process, an unbiased estimator of $\lambda$ would be equal to $n(x) / \operatorname{area}(B), n(x)$ is the number of points observed in area $B$.

In the context of maritime risk assessment, the intensity of a point process such as serious collisions will vary from one location to another. In other words, $X$ is more likely to be an inhomogeneous process. The expected number of points falling into a small area du around a location of interest $u$ is equal to $\lambda(u)$. Then the intensity of the process can be described as a function whereby the number of points in $B$ will be:

$$
E[N(X \cap B)]=\int_{B} \lambda(u) d u \text { for any sub-region } B
$$

Standard statistical regression techniques are available to model the intensity function in order to better understand a spatial point process. For example, an inhomogenous spatial Poisson model can be used to model intensity as a loglinear function of traffic density:

$$
\lambda(u)=\exp \left(\beta_{0}+\beta_{1} Z(u)\right)
$$

where $\beta_{0}, \beta_{1}$ are parameters and $Z(u)$ is a spatial covariate representing the traffic at location $u$. Traffic at location $u$ might be defined in any number of ways including the density of vessel movements or frequency of collision candidates (Montewka et al. 2011, Hansen, 2007). Similarly, the model might be extended to investigate additional spatial covariates considered to be associated with increased likelihood of risk exposure. For example, dynamic environmental factors such as wind, waves, or currents, or known geographical features such as areas identified as being difficult to navigate.

Unfortunately, datasets containing the data required to fit this type of model are limited in shipping, in particular incident data are often of adequate quality. Obtaining detailed historical data on the nature of maritime accidents such as precise location of the events is very challenging. In most 
instances, chains of events are not recorded properly. Another limitation with applying the standard spatial model here is that, as defined above, they do not allow for risk to vary at the ship level. For these reasons, an alternative framework is proposed that still facilitates investigation of the intensity function and follows the general aim of (3)

\subsection{Alternative approach and future additions}

To account for safety quality at the ship level, we propose to estimate various types and degrees of seriousness of incidents based on global incident data. For an individual ship $(i)$ that enters the area of interest within the time frame of interest, we estimate the underlying risk $\left(p_{i}\right)$ (a proxy for safety quality of a vessel) for a number of different endpoints such as for instance collisions or groundings. One could consider various methods such as logit models, survival analysis, Bayesian networks, classification trees etc. (Knapp, 2011, Bijwaard and Knapp, 2009, Hansen, 2007) for this purpose. In our approach, we quantify the safety quality of a vessel which combines human error and technical failures given a set of ship specific explanatory factors (age, size, ship type, flag, classification society, beneficial ownership, safety management company (the Document of Compliance Company), changes of ship particulars and ship management over time, past inspection and incident history).

Every time a vessel enters an area of interest, e.g. the Australian Exclusive Economic Zone (EEZ), a set of probabilities $p_{i}$ can be estimated dynamically for the vessel and attached to location specific parameters such as longitude and latitude. This allows ship-level risk to depend on location and derive risk at location $u$. One way to derive this is to define a spatial covariate that captures total risk exposure due to traffic generally as $\boldsymbol{Z}$ and partition annual ship-level risk according to the relative traffic exposure at each location of interest, such as

$$
p_{i}(u)=p_{i} \frac{Z_{i}(u)}{Z_{\mathrm{i}}}
$$

where $Z_{i}(u)$ represents the spatial process describing movement of ship $i$ at any location $u \in W$ (e.g. within the EEZ) and $Z_{i}$ is the total vessel activity for ship $i$ in a single year. $Z$ may be described as operation time at sea in days and then areas with high traffic exposure would correspond to areas where vessels spent prolonged periods of time. In principle, there will be many other metrics that can be considered for $Z$ such as distance travelled, frequency of collision candidates, etc. 
Following the same motivation as before, we are interested in understanding how the intensity might vary from location to location and attempt to derive the number of expected incidents in subregion $B$ aggregated across all ships. One way of doing this is to define a grid $G$ that partitions $W$ into disjoint measurable sets $S_{j} \subset W$ of area $a_{j}$ for $j=1, \ldots, J$. The typical pixel grid is a rectangular array in which all pixels have the same area; pixels of unequal area will be necessary for the current application as the surface is curved. We are interested in estimating $N_{j}=N\left(X \cap S_{j}\right)$ the number of points expected to fall in pixel $S_{j}$ and from (5) we can calculate the probability that an event for individual ship $i$ falls into $S_{j}$, as $p_{i j}=E\left[Y_{i j}\right]$ where $Y_{i j}=I\left\{Y_{i} \cap S_{j}\right\}$ the indicator for an incident and we can now derive

$$
N_{j}=N\left(X \cap S_{j}\right)=\sum_{i} p_{i j}
$$

Furthermore, calculations above are amended by replacing $Z_{i}$ with the expected total annual activity for the corresponding ship type, $E\left[Z_{\ell}\right]$, which can be derived from global traffic data. Defined in this way, we are implicitly assuming that total risk for the vessels observed in the study region is the same global ship-level risk but scaled (down) according to the fraction of traffic exposure. In other words, we are defining a baseline risk for a given level and type of traffic based on incident models estimated on global incident data. In principle, (5) may be extended to accommodate other spatial covariate risk layers as indicated earlier. For example, in the case increased risk associated with powered grounding when traversing an area with poor under-keel clearance. Conversely, the risk might be adjusted in the presence of a risk control option operating in a particular area (or across an entire sub-region).

Additional research is currently being carried out in order to develop a model that can simulate vessel trajectories for large drifting vessels. Another area of active research deals with the quantification of the effect of risk control options by means of expert knowledge. In terms of endpoints, one could also integrate sensitive coastal areas (Carey et al., 2014) to account for ecological, social, cultural or economic damages due to pollution from ships beyond the monetary value estimated for damages which can be quantified in monetary terms. Similar to risk control options, this area is dominated by expert knowledge and associated challenges (Hayes, 2011). In principle, each component can be incorporated into the overall framework by adding more terms to (5). In the pilot presented later, we demonstrate this for one selected risk control option (inspections which applies to all ships) as well as the quantification of an alternative endpoint (monetary value at risk). 


\subsection{Uncertainties}

Before we can develop a method that can adequately characterise uncertainty, it would be important to further examine the use of traffic data. For example, rules for deriving traffic intensity and patterns for a given period should be further refined and then more efficiently coded to process the raw data and reliably derive the traffic metrics of interest. Moreover, once such tools are readily available, it would be interesting to analyse variation in year-on-year traffic. For example, whilst it is possible that the main shipping routes do not markedly change, it is very plausible that the relative intensity between shipping routes will vary from year to year and possible that new routes may open. It is therefore not surprising to read elsewhere that the dynamic nature of the process underlying maritime traffic is complex. (Vanek O et al., 2012).

Developing a simple and reliable method to forecast traffic would also be a required input for predicting risk exposure in the future. The assumption inherently made by many maritime risk assessments involves a linear relationship between change in traffic over time and risk of groundings and increase according to square of the change for collisions (Cockcroft AN, 1976). These assumptions might be further investigated by re-analysis of existing time series data.

An important consideration is around uncertainty. One problem with current approaches in maritime risk assessment is that the decision maker is led to believe that the results are definitive and in no way uncertain. This is obviously not true. Firstly, we are assuming the simplified model, as outlined above, provides an adequate representation of reality. A method for understanding uncertainty is required. The general concept of uncertainty in assessment of risk assessment is well established in the literature (Hayes, 2011) including the various types of uncertainties. The majority of approaches to maritime risk assessment do not attempt to quantify uncertainty (Merrick, 2006, Eide et al., 2007). Furthermore, in the few cases where uncertainty intervals are provided, authors take great care to acknowledge the wide range of sources and assumptions made and highlight that complete quantification of uncertainty is not possible.

Uncertainties arise from input data, parameter estimates, as well as simplifications and assumptions used in the modelling approach. If qualitative methods are used based on subjective judgement, additional challenges arise. These could be due to different perceptions, beliefs and experiences and cognitive biases (Pidgeon et al. 1992, Rohrmann 1994, Kahneman \& Tversky 1984, Fischhoff et al. 1977). Hence, several sources of uncertainty that might be considered under the framework presented earlier are as follows:

- traffic intensity and patterns within the area of interest 
- $\quad$ ship specific incident risk and changes of risk profiles over time

- environmental conditions, e.g. currents, wind and wave data at a low grid resolution

- parameters associated with impact of environmental conditions on incident risk

- effect of control options, either at the macro level such as inspections or audits or at subregional level such as pilotage, vessel traffic services or under-keel clearance

- environmental sensitivities such as damages to the ecology, social, cultural or economic parameters

- uncertainties associates with the elicitation of expert knowledge

Following a similar approach to Merrick (2006), one way to adapt the proposed method and incorporate uncertainty, is to implement a dynamic multi-year Monte Carlo simulation. Firstly, a micro-level model for $Z(u)$, the maritime traffic system within an area of interest, would need to be developed. Simulation of $Z(u)$ could then be carried out by incorporating vessel movements (and potentially also incorporate a separate process for environmental conditions). For each simulated time period, ship level probabilities, $p_{i}$, would also be sampled from the global ship level incident model and applied to the ship tracks generated at each step of the simulation. A multi-year system simulation would be used to count the number of incidents and store the results of each event, i.e. type of ship, location of incident, as well as any other information relating to input data from the model. By repeatedly running the simulation according to the uncertainty described by data and parameters mentioned earlier, uncertainty can be then propagated through the whole system.

Note that developing a traffic model for $Z(u)$ is not the same as an alternative analysis that attempts describe the observed output of $Z(u)$, for example at different snapshots over time. Developing a traffic model would be substantially more complex to implement (Vanek, 2012) whereas the latter would entail learning about the observed traffic during selected periods. Both kinds of approaches would be invaluable to maritime administrations. As pointed out by Merrick (2006), the use of such a system simulation would also allow for the system-wide evaluation of risk reduction and risk mitigation effects potentially associated with the implementation of particular risk intervention measures. On the other hand, the alternative analysis would facilitate developing a time series model and provide a way to make forecasts for traffic.

Based on the theoretical framework presented, we present a proof of concept of the idea which combines the first two risk layers - ship specific risk and vessel traffic densities. We base this part on the outcome of a pilot study performed in conjunction with the Australian Maritime Safety Authority (AMSA) with the area of interest being the Australian Exclusive Economic Zone (EZZ). 
We provide examples on how estimates can be presented either for real time applications or longer term strategic planning routines.

\section{Proof of concept for integrating ship specific risk with vessel traffic densities}

\subsection{Data and risk components used}

Table 1 provides an overview of the data used with their data sources. Vessel traffic data was used from AMSA's Craft Tracking System (CTS) which combines all movement of crafts including ships. The CTS system also performs an automated identity check and verifies positions. As mentioned earlier, incident type models are based on Knapp (2006, 2011) by means of binary logistic regression that allow the estimation of various types and degrees of seriousness of incidents for which a subset was selected and applied (collisions, groundings, main engine failures, steering gear failures and anchor and mooring failures). The incident type probabilities are to be interpreted as yearly probabilities but had to be calibrated using global incident rates (for the years 2006 to 2010) to compensate for a slight overestimation of the probabilities due to the creation of the underlying matrix to estimate the models. The underlying incident data comes from four different sources and was manually reclassified using IMO definitions for seriousness (IMO, 2000). While we do not present this here in the high level results, we can distinguish risk by main ship type such as general cargo, dry bulk, container, tanker, passenger vessels and other ship types.

Table 1: Summary of data used

\begin{tabular}{|l|l|l|}
\hline Data & Time frame, nr. of observations & Use of data \\
\hline Vessel positions & $27 / 05 / 2012$ to 31/05/2013, & Risk exposure estimation \\
AMSA & $89,834,785$ initial observations & \\
\hline $\begin{array}{l}\text { Global incident data } \\
\text { Source: } A M S A, \text { IHSF, IMO, LMIU }\end{array}$ & $\begin{array}{l}01 / 01 / 2006 \text { to 31/12/2010 } \\
19,740 \text { observations }\end{array}$ & $\begin{array}{l}\text { Incident type models } \\
\text { Model validation }\end{array}$ \\
\hline $\begin{array}{l}\text { Regional incident data } \\
\text { Source: } \text { AMSA, Nexus }\end{array}$ & $02 / 01 / 1995$ to 01/06/2013, & Model validation \\
\hline $\begin{array}{l}\text { World fleet data at ship level } \\
\text { Source: } I H S \text {-Fairplay }\end{array}$ & 2006 to 2010 and 2012 & $\begin{array}{l}\text { Incident type models } \\
\text { Ship type classifications } \\
\text { Calibration factors }\end{array}$ \\
\hline $\begin{array}{l}\text { Nautical miles travelled } \\
\text { Source: } I M O, \text { LMIU }\end{array}$ & & Calibration factors \\
\hline
\end{tabular}

Besides the incident type models as proxy for safety quality, we apply a methodology developed by Knapp et al. (2011) to estimate the effect of a port state control inspection (PSC) in decreasing incident type risk based on survival analysis where ship life cycles were analyzed. This is to demonstrate how risk control options available to a maritime administration can be incorporated 
into the general framework described earlier as presented in Figure 1. The inspection effect becomes the ratio of survival probabilities with PSC to survival probability without PSC and is measured at the individual ship level. The effect of PSC inspections are larger on substandard ships compared to good quality ships.

To explore the possibility to express risk exposure in monetary terms, the methodology to estimate the monetary value at risk (MVR) developed by Heij et al. (2013) was used. MVR is a combination of the total insured value (TIV) of a vessel of five damage types (hull and machinery, life, oil pollution, third party liability and damages to cargo). The TIV values at the individual ship level are adjusted by a set of damage type probabilities for each vessel. MVR is best to be interpreted as an approximation of risk exposure in monetary terms to be mitigated by a maritime administration AMSA while in reality this figure will be higher since damages to the marine ecology or cultural heritage cannot be quantified in monetary terms.

\subsection{Derivation of vessel traffic parameters}

Analysis of the CTS data was required in order to derive the shipping traffic pattern that forms the basis of the risk exposure methodology. The CTS dataset has to undergo a data cleaning routine in order to identify potential data errors and/or illogical values. This was necessary in order to obtain a dataset containing a clean and consistent set of ship tracks within the Australian EEZ.

The next step involves placing a spatial grid overlay with a 10' by 10' unit grid (approximately 100 square nautical miles) onto the area of interest and calculating total traffic observed within each grid. The grid resolution used at this step can be modified. Traffic parameters were combined together with individual ship level probabilities in order to derive a risk layer as follows. For each vessel, the corresponding set of ship-level incident probabilities were calibrated by the ratio of distance travelled in the EEZ divided by the average annual total ship miles expected for that vessel type (IMO, 2009). The approach is then carried out for all vessels based on the individual ship-level data. Note that there is marked variation between individual vessels in both ship miles travelled as well as underlying annual risk.

Similar calculations can be carried out based on a different traffic metric, e.g. operation time as defined by days at sea was also evaluated. Furthermore, in principle, there would be many different options for deriving individual ship-level calibration factors including more geometrically defined constructs such as number of collision encounters (also referred to as collision candidates). Such an approach would be more in-line with other conventional maritime risk assessment methods but was 
not considered further. In the future, we aim to introduce and evaluate a formal way of incorporating collision candidates into our framework. This step however involves extensive programming and is highly dependent on adequacy of the ship track data. Our approach outlined above provides a first step in combining two components of a potentially multi-layered risk assessment approach.

\subsection{Results and model validations}

The results presented here should provide an overview of the type of endpoints that can be considered as well as how they are visualized for either real time application or medium/long term strategic planning. For real time application, the ship specific probabilities can be linked to traffic data and displayed in real time (Heij et al., 2013). If a high risk vessel approaches a high risk area or high traffic density area, automatic alerts could be triggered in operational systems. For medium to long term planning aspects, a maritime administration would be interested in estimating risk within its exposure area and to predict risk exposure given future traffic scenarios.

Table 2 presents an example where we compare the expected number of incidents to that observed. These results are based on 53 million observations of vessel positions, 43.6 million of nm travelled and risk profiles of 8,900 individual ships. It is worth noting that observed incidents are not accurate and should be treated with caution if compared by region due to the quality issues associated with incident data. Around $50 \%$ of the observations lack coordinates (lat/long) and it is difficult to associate them with a particular area. For ease of comparison, Table 2 presents total figures but the proposed methodology presented earlier can be applied to any spatial resolution of interest, e.g. Great Barrier Reef, or a particular shipping channel etc.

Table 2: Expected numbers of incidents in the Australian EEZ (yearly)

\begin{tabular}{|ll|r|r|r|r|}
\hline \multirow{2}{*}{ Results by year } & Risk control & \multicolumn{4}{|c|}{ Incident type (VSS) } \\
\cline { 3 - 6 } & Options & Collisions & Powered groundings & Drift groundings & Total \\
\hline Observed (historical data) & with all RCO $\dagger$ & 1.80 & 2.00 & 4.60 & $\mathbf{8 . 4 0}$ \\
Expected & with PSC & 3.33 & 2.93 & 4.51 & $\mathbf{1 0 . 7 7}$ \\
& w/o PSC & 3.74 & 3.32 & 5.09 & $\mathbf{1 2 . 1 5}$ \\
\hline
\end{tabular}

Notes: VSS = very serious and serious incidents, $R C O=$ risk control options, ship types of 'unknown' category were removed from these calculations; †observed incident data within the EEZ reflect any RCOs that were in operation during that period

A different endpoint measurement would be expressing risk exposure in monetary terms. We have explored this and at this stage, can present this for the Australian EEZ as one estimate. We use the monetary value at risk (MVR) as proxy to potential damages due to incidents except damage types which cannot be quantified (e.g. damages to the ecology, cultural heritages). Based on 43.6 million 
of $\mathrm{nm}$ travelled and the individual risk profiles of the vessels that traded in the Australian EEZ over the course of a year, MVR for very serious incidents is estimated to be AUD 109.6 million (without PSC effect) and AUD 96.4 million (with PSC effect). If besides very serious incidents, serious incidents are considered, then MVR will increase respectively. The developed methodology can accommodate various degrees of seriousness depending on the need of coastal or port states. Location specific or regional aggregation including the best visualization of MVR needs further development and is therefore not presented here.

Spatial visualization of estimates is important for policy makers in order to understand the spatial dimension to risk exposure and to identity hot spots. Based on the proposed methodology, the results for expected numbers of incidents can be visualized spatially with flexible resolution (refer to Figures 2 to 4 for a resolution of approximately 10nm and Figure 5 for examples of $1 \mathrm{~nm}$ ). The expected numbers are calculated for each grid based on the safety quality of the ships that travelled in this area and the vessel traffic density which determines the ship specific risk exposure. The categories used to classify the risk levels are provided in Table 3. Note that the thresholds were based on providing a roughly equal number of grid areas (e.g. 200 for collision risk) for the 'moderate', 'high', and 'very high' categories. All of the grid areas remaining from the 31,835 grids defining the EEZ study area were then allocated to very low and low risk. The expected numbers of events appear very low as the associated area size (of a grid) is also very small (recall it is $\sim 100$ $\left.\mathrm{nm}^{2}\right)$.

Table 3: Risk categories used for visualization

\begin{tabular}{|l|l|l|}
\hline Expected number & Category & Interpretation \\
\hline$<0.0001$ & Very Low & $\begin{array}{l}\text { Plausible minimum counterfactual } \\
\text { for EEZ is } 0.5 \text { under the Very Low risk category }\end{array}$ \\
\hline$(0.0001-0.001]$ & Low & 6 -60 fold higher \\
\hline$(0.001-0.0015]$ & Moderate & $60-100$ fold higher \\
\hline$(0.0015-0.002]$ & High & $100-130$ fold higher \\
\hline$>0.002$ & Very High & Greater than 130 fold higher \\
\hline
\end{tabular}

The following interpretation is put forward to assist with comparing risk categories. Consider a plausible minimum counterfactual risk level whereby the entire study region was very low risk. Under this scenario, the overall expected number of (say) collisions annually would be approximately equal to 0.5 . Each of the risk categories above 'very low' risk can then be more easily compared. For example, 'high risk' is more than 130-fold higher than the baseline hypothetical counterfactual of 0.5 events per year. The notion of counterfactual exposure distributions is touched on further in concluding remarks. 
In order to further gauge the face validity of our approach, the same risk maps also include an overlay of historical maritime incidents, all available recorded incidents occurring from January 1995 to June 2013. Note that this provides a comparison between outcomes that occurred in the past and estimated risk based on traffic intensity and patterns towards the end of this time series. A shorter time period (e.g. last 5 years or 10 years) would have been preferable but around $50 \%$ of the observations lack coordinates (lat/long). Therefore, a longer time period was needed.

Note that Figure 4 is more difficult to interpret since we use proxy variables (main engine failures, steering gear failures and anchor and mooring failures) to approximate drift groundings and where it is equally difficult to distinguish between drift groundings and powered groundings in the historical data. Despite all of these limitations, it is still of interest to assess whether maritime incidents have occurred where we might expect them to occur based on a relatively simple risk metric. Here we assume that the individual ship risk profiles of vessels trading in Australia has not changed considerably over time. Furthermore, location specific environmental conditions are not considered yet in the analysis.

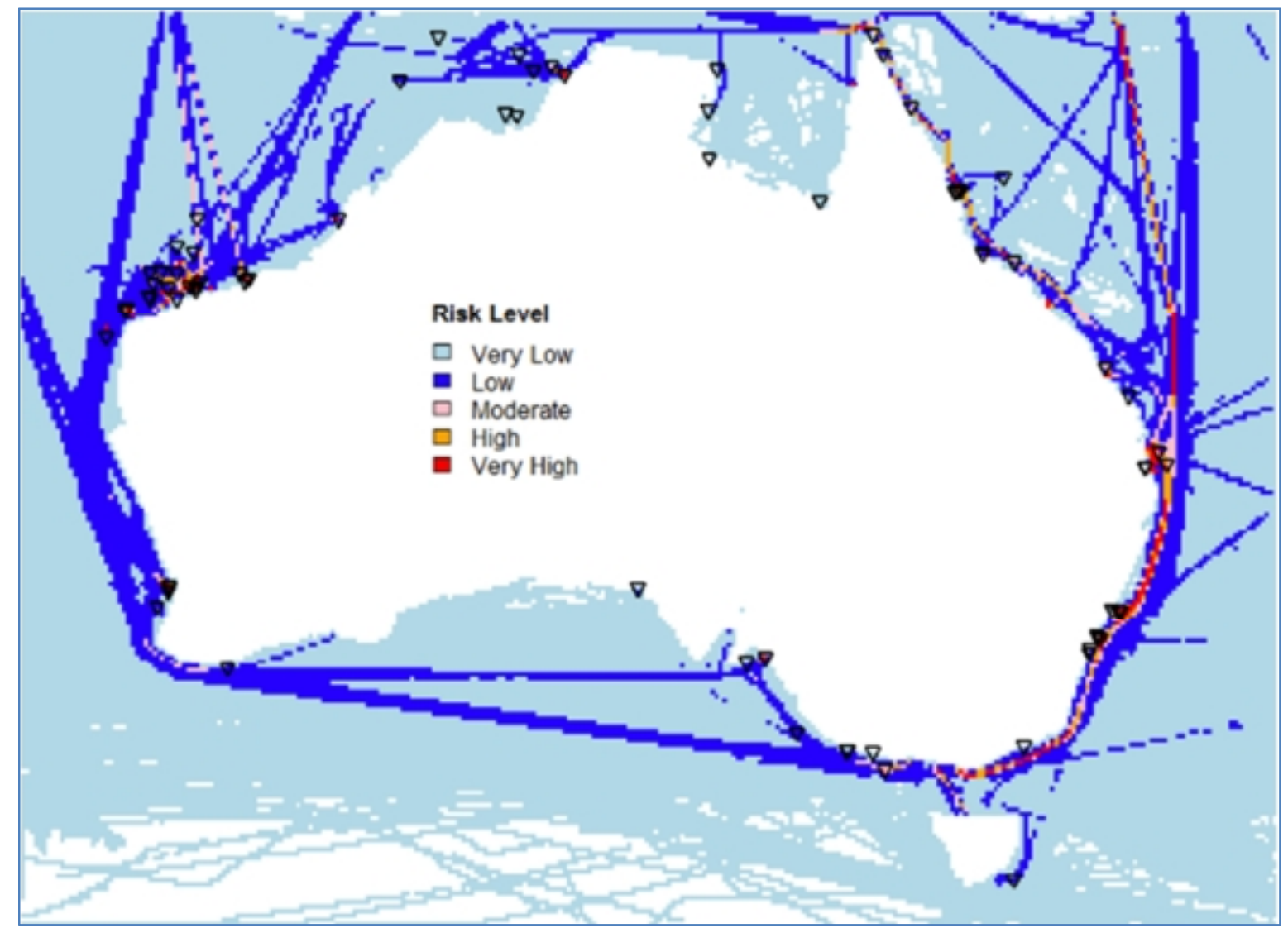

Figure 2: Observed collisions (any seriousness) during 1995 to June 2013 overlaid (as black triangles) onto the risk assessment for collisions (VSS) in the Australian EEZ, 10' resolution 


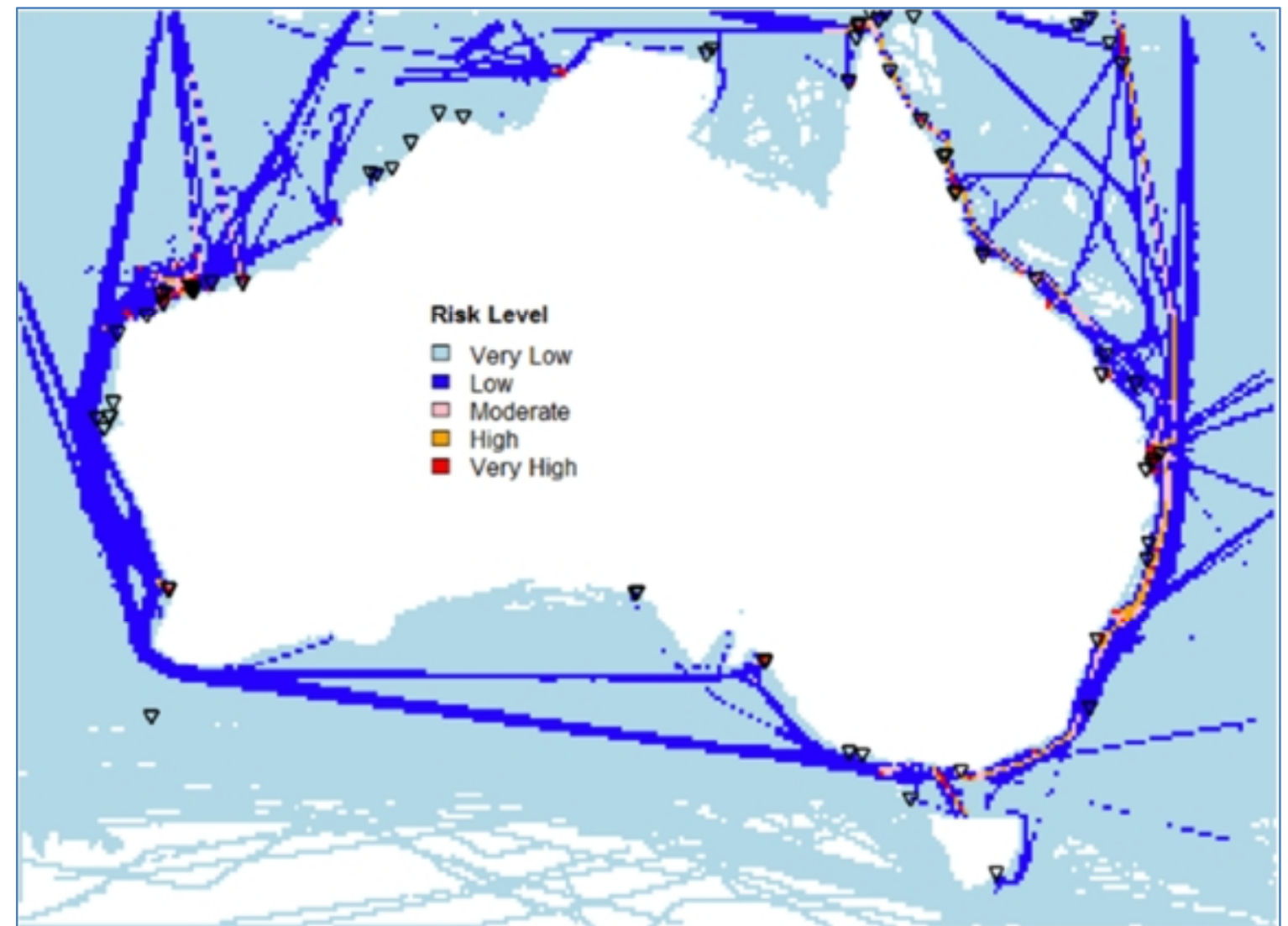

Figure 3: Observed powered groundings (any seriousness) during 1995 to June 2013 overlaid (as black triangles) onto the risk assessment for groundings (VSS) in the Australian EEZ, 10' resolution

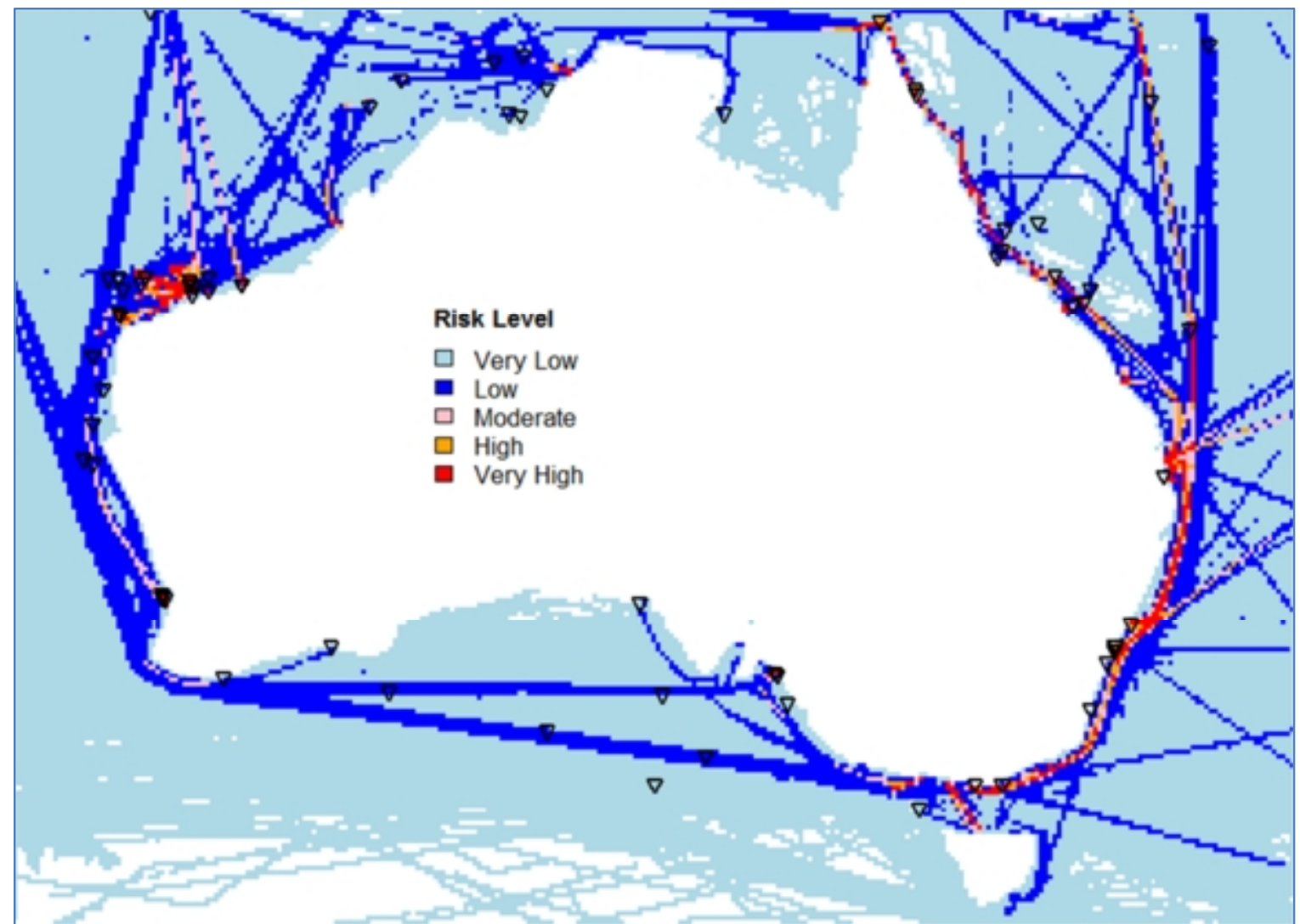

Figure 4: Observed drift groundings (any seriousness of main engine failures excluding stoppages, steering gear failures and anchor and mooring failures during 1995 to June 2013) overlaid (as black triangles) onto the risk assessment for drift groundings (VSS) in the Australian EEZ, 10' resolution 

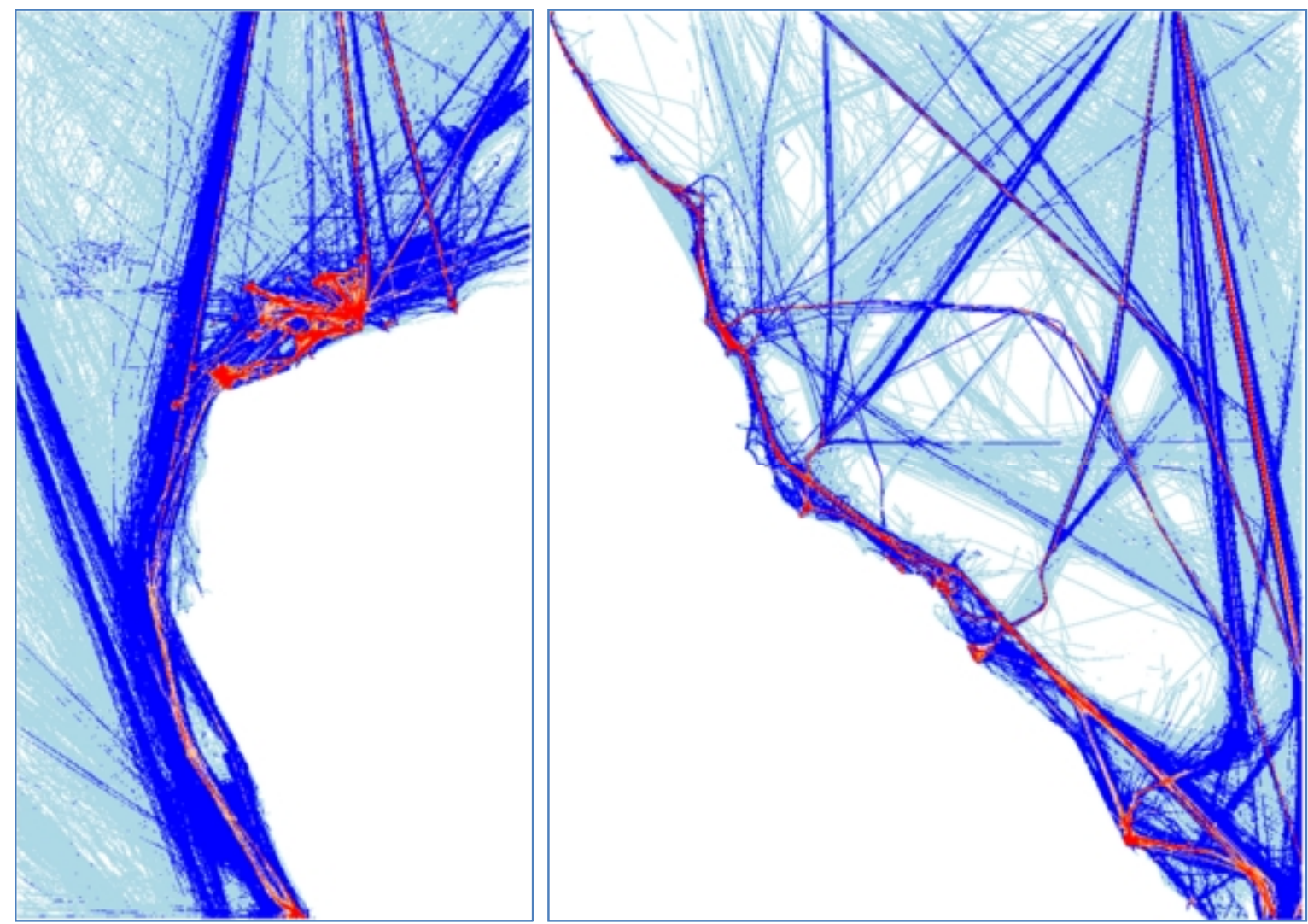

Figure 5: Example for expected Nr of drift groundings (VSS) in the North West (left) and the Great Barrier Reef (right) area, 1' resolution

Notwithstanding this limitation, each of the risk overlays above suggests a reasonable general level of concordance between locations considered to be high or very high risk and position of historical data. This can be refined in the future if incident data quality improves. For example, Figure 2 presents our risk assessment for collisions in 2012-2013 and it is apparent that approximately half of the observed collisions (of any seriousness) occurred at, or very near, to a high or very high risk grid area. It is also of interest to note the two main sub-regions expected to have higher risk also experienced clears clusters of collision related events.

\section{Conclusions and future research}

The proposed conceptual framework provides a relatively straight forward approach to maritime risk assessment at the macro level. The major difference to other approaches is that we divide total risk exposure into risk layers which can be treated individually or in combined format. In such, the proposed approach allows the combination of the risk layers with flexible spatial resolution. One of its advantages is that it starts at the individual ship level in an attempt to quantify safety quality since risk is not homogenous across ships or areas. Combined with vessel traffic densities, this methodology can be implemented into traffic systems in either real time mode or used to estimate 
risk exposure expressed as various endpoints (e.g. estimated number of incidents, monetary value at risk).

Several extensions of this application include: to improve estimation of risk at different spatial scales; to assess risk over time; and to predict risk in the future given risk control scenarios. In theory, risk can be calculated for each of the main shipping routes in the future or for areas of interest to a maritime administration. Most important layer extensions to the framework presented here are therefore associated with location specific risk parameters such as risk control options or underlying environmental conditions (wind, waves and currents). Both risk layer components are currently being developed.

We also considered uncertainties and present various sources which would need to be considered if integrated into the general framework and more research is currently also dealing with this aspect. We aim to further assess all sources of uncertainty either stemming from input data or arising from simplifications and assumptions used in the modelling approach. One of the key next future steps will be to address how uncertainty is best handled here although, in reality, we recognise that a complete assessment of uncertainty will never be truly attainable.

The taxonomy of counterfactual exposure distributions is used frequently in other application areas (Murray CJL and Lopez AD, 1999) and would also be very useful within the context considered here to further assist with interpretation of results from maritime risk assessment. For example, theoretical minimum risk is the exposure distribution that would result in the lowest total risk, i.e. zero incidents, irrespective of whether currently attainable in practice. Plausible minimum risk refers to a distribution which is imaginable and feasible and is one that has been observed in particular sub-regions. Several other counterfactual distributions will be considered in the proposed framework here including the cost effective minimum risk in order to consider the cost of exposure reduction for a given set of RCOs. These definitions can be set according to the policy maker's perception of risk.

It is important to consider that risk assessment should allow for multiple approaches as each one will have its own strengths and limitations and will facilitate addressing different questions of interest of a maritime operation such as operational real-time monitoring of vessel traffic or medium or longer term strategic planning. Different approaches may be complementary, e.g. when a microlevel (mechanistic) model provides a better approach to quantify the effect of an intervention in a 
specific area while macro-level models allow real time applications for larger areas or strategic planning exercises.

\section{Acknowledgements}

The authors wish to thank the Australian Maritime Safety Authority, IHS-Maritime, LMIU and IMO for providing the necessary incident, fleet and traffic data. Furthermore the authors would like to thank Jillian Carson-Jackson from AMSA for her input.

\section{References}

Badderly A (2010), Analysing spatial point patterns in R - Workshop Notes, CSIRO and University of Western Australia

Bijwaard G and Knapp S (2009), Analysis of Ship Life Cycles - The Impact of Economic Cycles and Ship Inspections, Marine Policy, 33(2): 350-369

Carey J, Knapp S. and Irving P (2014), Assessing ecological sensitivities of marine assets to oil spill by means of expert knowledge, Econometric Institute Report 2014-13, Erasmus University

Cockcroft AN (1976), Statistics of collisions at sea, Collision Risk Reduction, 29: 215-231

de Smith MJ, Goodchild MF and Longley PA (2013), Geospatial Analysis: A comprehensive guide to principles, techniques and software tools www.spatialanalysisonline.com

Eide MS, Endresen O, Brude OW, Brett PO, Breivik O, Ellingsen IH, Roang K and Hauge J (2007), "Prevention of oil spill from shipping by modelling of dynamic risk." Marine Pollution Bulletin 54.10: 1619-1633.

Fischoff B, Slovia P and Lichtenstein S (1977), Knowning with certainty: the appropriateness of extreme confidence. Journal of Experimental Psychology: Human Perception and Performance. 3: 552-564.

Hansen PH (2007), IWRAP II, Working Document, Basic Modelling Principles for Prediction of Collision and Grounding Frequencies, Technical University of Denmark.

Hayes KR (2011), Uncertainty and uncertainty analysis methods. ACERA Report Number: EP102467. Australian Centre of Excellence for Risk Analysis, Melbourne

Heij C, Bijwaard G and Knapp S (2011), Ship Inspection Strategies: Effects on Maritime Safety and Environmental Protection, Transportation Research Part D, 16, 42-48

Heij C and Knapp S (2012), Evaluation of safety and environmental risk at individual ship and company level, Transportation Research Part D 17 228-236

Heij C, Knapp S, Henderson R and Kleverlaan E (2013), Ship incident risk around the heritage areas of Tubbataha and Banc d'Arguin, Transportation Research Part D 25: 77-83

Heij C and Knapp S (2014), Effects of wind strength and wave height on ship incident risk: regional trends and seasonality, Econometric Institute Report 2014-15, Erasmus University

IMO (2000), MSC/Circ. 953, MEPC/Circ. 372, Reports on Marine Casualties and Incidents, Revised harmonized reporting procedures, adopted $14^{\text {th }}$ December 2000, IMO, London. 
IMO (2009), Second IMO GHG Study 2009, International Maritime Organization (IMO), London, UK, April 2009; Buhaug, Ø., Corbett, J.J., Endresen, Ø., Eyring, V., Faber, J., Hanayama, S., Lee, D.S., Lee, D., Lindstad, H.,Markowska, A.Z., Mjelde, A., Nelissen, D., Nilsen, J., Pålsson, C., Winebrake, J.J., Wu, W., Yoshida, K

Kahneman D and Tversky A (1984), Choices, values, and frames. American Psychologist. 39: 341350.

Knapp, S (2006), The Econometrics of Maritime Safety - Recommendations to improve safety at sea, Doctoral Thesis, Erasmus University Rotterdam

Knapp S, (2011), Integrated risk estimation methodology and implementation aspects (Main Report AMSA); Reference Nr. TRIM 2010/860, 2011-1a (Main Report AMSA)

Knapp S, Bijwaard G and Heij C (2011), Estimated Incident Cost Savings in Shipping due to Inspections, Accident Analysis and Prevention, 43: 1532-1539

Merrick JRW and van Dorp R (2006), Speaking the Truth in Maritime Risk Assessment, Risk Analysis, Risk Analysis vol. 26 (1): 223-237

Montewka J, Goerlandt F, Hanninen M, Ylitalo J and Seppala T (2011), Algorithm development and documentation, Study of algorithm development using data mining, EfficientSea Project, www.efficiensea.org

Murray CJL and Lopez AD (1999), On the Comparative Quantification of Health Risks: Lessons from the Global Burden of Disease, Epidemiology 10: 594-605

Pidgeon N, Hood C, Jones C, Turner B and Gibson R. (1992), Risk perception, Risk Analysis, Perception and Management. Report of the Royal Society Study Group. The Royal Society, London. UK Ch. 5.

Rohrmann B (1994), Ris perception of different societal groups: Australin findings and crossnational comparisons. Australian Journal of Psychology. 46: 150-163.

UNCTAD, 2010, 2011, Review of Maritime Transport 2010, 2011, UNCTAD, Geneva

Vanek O, Jakob M, Hrstka O and Pechoucek M (2012), Using Multi-agent Simulation to Improve the Security of Maritime Transit, Lecture Notes in Computer Science Volume 7124, 2012, pp 4458 